\title{
Some Bounds of the Zeros of Polynomials Based on the QR-Decomposition and the LU-Decomposition of the Frobenius Companion Matrix
}

\author{
Ammar S. Issa ${ }^{1} \&$ Omar M. Bdair ${ }^{1}$ \\ ${ }^{1}$ Department of Applied Sciences, Faculty of Engineering Technology, Al Balqa Applied University, Amman, \\ Jordan \\ Correspondence: Omar M. Bdair, Department of Applied Sciences, Faculty of Engineering Technology, Al Balqa \\ Applied University, Amman, Jordan. E-mail: bdairmb@yahoo.com
}

Received: October 22, 2013 Accepted: November 30, 2013 Online Published: December 30, 2013

doi:10.5539/jmr.v6n1p33 URL: http://dx.doi.org/10.5539/jmr.v6n1p33

\begin{abstract}
In this paper, new bounds of zeros of polynomials of the monic polynomial of order $n$ will be introduced by applying famous matrix norms to the QR-decomposition of $C(p)$. The LU decomposition of $C(p)$ will be investigated and will be used to find more bounds of the zeros of $p(z)$.
\end{abstract}

Keywords: QR decomposition, LU decomposition, matrix norms, inequalities

MR Subject Classification: 42B20, 42B25

\section{Introduction}

Companion matrices play an important role in matrix theory, numerical analysis, and numerical linear algebra. Their importance comes from their role in canonical forms and their connection with the location of zeros of polynomials. Finding bounds for zeros of polynomials is an important and old issue to study. In this paper we will introduce a new bounds for the zeros of polynomials using decomposition of companion matrices and we will apply some of the well-known bounds to these decompositions.

Let

$$
C(p)=C=\left[\begin{array}{cccccc}
-a_{n} & -a_{n-1} & -a_{n-2} & \cdots & -a_{2} & -a_{1} \\
1 & 0 & 0 & \cdots & 0 & 0 \\
0 & 1 & 0 & \cdots & 0 & 0 \\
\vdots & \vdots & \vdots & \ddots & \vdots & \vdots \\
0 & 0 & 0 & \cdots & 0 & 0 \\
0 & 0 & 0 & \cdots & 1 & 0
\end{array}\right],
$$

be the Frobenius companion matrix corresponding to the complex monic polynomial $p(z)=z^{n}+a_{n} z^{n-1}+\cdots+$ $a_{2} z+a_{1}$, it is will known that the zeros of $p(z)$ and the eigenvalues of $C(p)$ are the same. For more details about the companion matrix and the complex monic polynomial one may refer to Horn and Johnson (1985). Also if $r(C)$ denote the spectral radius of $C$ then it can be shown that $r(C) \leq N(C)$, where $N($.$) is any matrix norm, see$ for example Bahatia (1997) or Horn and Johnson (1991). Moreover, geometry of polynomials and other details about the zeros of polynomials are explained in Marden (1969) or Kittaneh (2003). Kittaneh and Shabrawi (2007), established some bounds for the zeros of monic polynomial depending on some matrices norms.

Kittaneh and Shebrawi (2006) proved an important theorem about the $Q R$ decomposition. They introduced the $Q R$ decomposition for the companion matrix $C(p)$ corresponding to $p(z)$ of degree $n \geq 2$ with $a_{1} \neq 0$, They proved that $C=Q R$, where 


$$
Q=\left[\begin{array}{cccccc}
\frac{-a_{n}}{\mu_{0} \mu_{1}} & \frac{-a_{n-1}}{\mu_{1} \mu_{2}} & \frac{-a_{n-2}}{\mu_{2} \mu_{3}} & \cdots & \frac{-a_{2}}{\mu_{n-2} \mu_{n-1}} & \frac{-a_{1}}{\mu_{n-1} \mu_{n}} \\
\frac{\mu_{0}^{2}}{\mu_{0} \mu_{1}} & \frac{-a_{n-1} \bar{a}_{n}}{\mu_{1} \mu_{2}} & \frac{-a_{n-2} \bar{a}_{n}}{\mu_{2} \mu_{3}} & \cdots & \frac{-a_{2} \bar{a}_{n}}{\mu_{n-2} \mu_{n-1}} & \frac{-a_{1} \bar{a}_{n}}{\mu_{n-1} \mu_{n}} \\
0 & \frac{\mu_{1}^{2}}{\mu_{1} \mu_{2}} & \frac{-a_{n-2} \bar{a}_{n-1}}{\mu_{2} \mu_{3}} & \cdots & \frac{-a_{2} \bar{a}_{n-1}}{\mu_{n-2} \mu_{n-1}} & \frac{-a_{1} \bar{a}_{n-1}}{\mu_{n-1} \mu_{n}} \\
0 & 0 & \frac{\mu_{2}^{2}}{\mu_{2} \mu_{3}} & \cdots & \frac{-a_{2} \bar{a}_{n-2}}{\mu_{n-2} \mu_{n-1}} & \frac{-a_{1} \bar{a}_{n-2}}{\mu_{n-1} \mu_{n}} \\
0 & 0 & 0 & \cdots & \frac{-a_{2} \bar{a}_{n-3}}{\mu_{n-2} \mu_{n-1}} & \frac{-a_{1} \bar{a}_{n-3}}{\mu_{n-1} \mu_{n}} \\
\vdots & \vdots & \vdots & \ddots & \vdots & \vdots \\
0 & 0 & 0 & \cdots & \frac{-a_{2} \bar{a}_{3}}{\mu_{n-2} \mu_{n-1}} & \frac{-a_{1} \bar{a}_{3}}{\mu_{n-1} \mu_{n}} \\
0 & 0 & 0 & \cdots & \frac{\mu_{n-2}^{2}}{\mu_{n-2} \mu_{n-1}} & \frac{-a_{1} \bar{a}_{2}}{\mu_{n-1} \mu_{n}}
\end{array}\right]
$$

is unitary, and

$$
R=\left[\begin{array}{cccccc}
\frac{\mu_{1}}{\mu_{0}} & \frac{a_{n-1} \bar{a}_{n}}{\mu_{0} \mu_{1}} & \frac{a_{n-2} \bar{a}_{n}}{\mu_{0} \mu_{1}} & \ldots & \frac{a_{2} \bar{a}_{n}}{\mu_{0} \mu_{1}} & \frac{a_{1} \bar{a}_{n}}{\mu_{0} \mu_{1}} \\
0 & \frac{\mu_{2}}{\mu_{1}} & \frac{a_{n-2} \bar{a}_{n-1}}{\mu_{1} \mu_{2}} & \ldots & \frac{a_{2} \bar{a}_{n-1}}{\mu_{1} \mu_{2}} & \frac{a_{1} \bar{a}_{n-1}}{\mu_{1} \mu_{2}} \\
0 & 0 & \frac{\mu_{3}}{\mu_{2}} & \ldots & \frac{a_{2} \bar{a}_{n-2}}{\mu_{2} \mu_{3}} & \frac{a_{1} \bar{a}_{n-2}}{\mu_{2} \mu_{3}} \\
\vdots & \vdots & \vdots & \ddots & \vdots & \vdots \\
0 & 0 & 0 & \ldots & \frac{\mu_{n-1}}{\mu_{n-2}} & \frac{a_{1} \bar{a}_{2}}{\mu_{n-1} \mu_{n-2}} \\
0 & 0 & 0 & \ldots & 0 & \frac{\mu_{n}}{\mu_{n-1}}
\end{array}\right]
$$

is upper triangular, with $\mu_{k}=\sqrt{1+\sum_{j=1}^{k}\left|a_{n-j+1}\right|^{2}}$ for $k=1,2, \ldots, n-1, \mu_{0}=1$, and $\mu_{n}=\left|a_{1}\right|$.

In this paper, we will introduce some new bounds for the zeros of polynomials depending on the $Q R$ and $L U$ decompositions of the companion matrix $C(p)$. The Euclidian norm $\left(\|\cdot\|_{2}\right)$, the maximum column norm $\left(\||| \cdot\|_{1}\right)$ and the maximum row sum norm $\left(\|\mid \cdot\|_{\infty}\right)$ will be applied for the above mentioned decompositions of the companion matrix to obtain the needed bounds.

\section{The LU Decomposition of the Frobenius Companion Matrix}

Issa (2009) established an $L U$ decomposition for the companion matrix corresponding to the complex monic polynomial $p(z)$ of degree $n \geq 2$ with $a_{i} \neq 0, i=1,2, \ldots, n$. Here, he proved that the companion matrix $C$ could be written as a product of $L$ (lower triangular matrix) and $U$ (upper triangular matrix).

Theorem 1 Let $C(p)$ be the Frobenius companion matrix corresponding to the complex monic polynomial $p(z)=$ $z^{n}+a_{n} z^{n-1}+a_{n-1} z^{n-2}+\cdots+a_{2} z+a_{1}$ of degree $n \geq 2$ with $a_{i} \neq 0, i=1,2, \ldots, n$. Then the $L U$ decomposition for $C$ is given by $C=L U$, where $L$ is given by the matrix 


$$
L=\left[\begin{array}{cccccccc}
1 & 0 & 0 & 0 & \cdots & 0 & 0 & 0 \\
-\frac{1}{a_{n}} & 1 & 0 & 0 & \cdots & 0 & 0 & 0 \\
0 & -\frac{a_{n}}{a_{n-1}} & 1 & 0 & \cdots & 0 & 0 & 0 \\
0 & 0 & -\frac{a_{n-1}}{a_{n-2}} & 1 & \cdots & 0 & 0 & 0 \\
0 & 0 & 0 & -\frac{a_{n-2}}{a_{n-3}} & \cdots & 0 & 0 & 0 \\
\vdots & \vdots & \vdots & \vdots & \ddots & \vdots & \vdots & \vdots \\
0 & 0 & 0 & 0 & \cdots & -\frac{a_{4}}{a_{3}} & 1 & 0 \\
0 & 0 & 0 & 0 & \cdots & 0 & -\frac{a_{3}}{a_{2}} & 1
\end{array}\right],
$$

and $U$ is given by the matrix

$$
U=\left[\begin{array}{cccccccc}
-a_{n} & -a_{n-1} & -a_{n-2} & -a_{n-3} & -a_{n-4} & \cdots & -a_{2} & -a_{1} \\
0 & -\frac{a_{n-1}}{a_{n}} & -\frac{a_{n-2}}{a_{n}} & -\frac{a_{n-3}}{a_{n}} & -\frac{a_{n-4}}{a_{n}} & \cdots & -\frac{a_{2}}{a_{n}} & -\frac{a_{1}}{a_{n}} \\
0 & 0 & -\frac{a_{n-2}}{a_{n-1}} & -\frac{a_{n-3}}{a_{n-1}} & -\frac{a_{n-4}}{a_{n-1}} & \cdots & -\frac{a_{2}}{a_{n-1}} & -\frac{a_{1}}{a_{n-1}} \\
0 & 0 & 0 & -\frac{a_{n-3}}{a_{n-2}} & -\frac{a_{n-4}}{a_{n-2}} & \cdots & -\frac{a_{2}}{a_{n-2}} & -\frac{a_{1}}{a_{n-2}} \\
0 & 0 & 0 & 0 & -\frac{a_{n-4}}{a_{n-3}} & \cdots & -\frac{a_{2}}{a_{n-3}} & -\frac{a_{1}}{a_{n-3}} \\
\vdots & \vdots & \vdots & \vdots & \vdots & \ddots & \vdots & \vdots \\
0 & 0 & 0 & 0 & 0 & \cdots & -\frac{a_{2}}{a_{3}} & -\frac{a_{1}}{a_{3}} \\
0 & 0 & 0 & 0 & 0 & \cdots & 0 & -\frac{a_{1}}{a_{2}}
\end{array}\right] .
$$

Proof. To compute the matrices $U=\left[u_{1}: u_{2}: \cdots: u_{n}\right]^{T}$ and $L=\left[l_{1}: l_{2}: \cdots: l_{n}\right]^{T}$, we apply the theorem mentioned in section 3.10 of Meyer (2000) which give us that $u_{j}=\left\{\begin{array}{l}r_{1}, j=1 \\ \frac{1}{a_{n}} u_{1}+r_{2}, j=2 \\ \frac{a_{n+3-j}}{a_{n+2-j}} u_{j-1}+r_{j}, j=3,4, \ldots, n,\end{array}\right.$ where $r_{j}$ and $u_{j}$ represent the $j^{\text {th }}$ rows of $C$ and $U$, respectively.

So we get that,

$$
\begin{gathered}
u_{1}=r_{1}=\left[\begin{array}{llllllll}
-a_{n} & -a_{n-1} & -a_{n-2} & -a_{n-3} & -a_{n-4} & \cdots & -a_{2} & -a_{1}
\end{array}\right] . \\
u_{2}=\frac{1}{a_{n}} u_{1}+r_{2}=\left[\begin{array}{llllllll}
0 & -\frac{a_{n-1}}{a_{n}} & -\frac{a_{n-2}}{a_{n}} & -\frac{a_{n-3}}{a_{n}} & -\frac{a_{n-4}}{a_{n}} & \cdots & -\frac{a_{2}}{a_{n}} & -\frac{a_{1}}{a_{n}}
\end{array}\right] . \\
u_{3}=\frac{a_{n}}{a_{n-1}} u_{2}+r_{3}=\left[\begin{array}{llllllll}
0 & 0 & -\frac{a_{n-2}}{a_{n-1}} & -\frac{a_{n-3}}{a_{n-1}} & -\frac{a_{n-4}}{a_{n-1}} & \cdots & -\frac{a_{2}}{a_{n-1}} & -\frac{a_{1}}{a_{n-1}}
\end{array}\right] . \\
u_{4}=\frac{a_{n-1}}{a_{n-2}} u_{3}+r_{4}=\left[\begin{array}{llllllll}
0 & 0 & 0 & -\frac{a_{n-3}}{a_{n-2}} & -\frac{a_{n-4}}{a_{n-2}} & \cdots & -\frac{a_{2}}{a_{n-2}} & -\frac{a_{1}}{a_{n-2}}
\end{array}\right] . \\
u_{5}=\frac{a_{n-2}}{a_{n-3}} u_{4}+r_{5}=\left[\begin{array}{llllllll}
0 & 0 & 0 & 0 & -\frac{a_{n-4}}{a_{n-3}} & \cdots & -\frac{a_{2}}{a_{n-3}} & -\frac{a_{1}}{a_{n-3}}
\end{array}\right] .
\end{gathered}
$$

Resuming like this until we get

$$
u_{n-1}=\frac{a_{4}}{a_{3}} u_{n-2}+r_{n-1}=\left[\begin{array}{cccccccc}
0 & 0 & 0 & 0 & 0 & \cdots & -\frac{a_{2}}{a_{3}} & -\frac{a_{1}}{a_{3}}
\end{array}\right] .
$$




$$
u_{n}=\frac{a_{3}}{a_{2}} u_{n-1}+r_{n}=\left[\begin{array}{llllllll}
0 & 0 & 0 & 0 & 0 & \cdots & 0 & -\frac{a_{1}}{a_{2}}
\end{array}\right] .
$$

Consequently, the upper triangular matrix

$$
U=\left[\begin{array}{cccccccc}
-a_{n} & -a_{n-1} & -a_{n-2} & -a_{n-3} & -a_{n-4} & \cdots & -a_{2} & -a_{1} \\
0 & -\frac{a_{n-1}}{a_{n}} & -\frac{a_{n-2}}{a_{n}} & -\frac{a_{n-3}}{a_{n}} & -\frac{a_{n-4}}{a_{n}} & \cdots & -\frac{a_{2}}{a_{n}} & -\frac{a_{1}}{a_{n}} \\
0 & 0 & -\frac{a_{n-2}}{a_{n-1}} & -\frac{a_{n-3}}{a_{n-1}} & -\frac{a_{n-4}}{a_{n-1}} & \cdots & -\frac{a_{2}}{a_{n-1}} & -\frac{a_{1}}{a_{n-1}} \\
0 & 0 & 0 & -\frac{a_{n-3}}{a_{n-2}} & -\frac{a_{n-4}}{a_{n-2}} & \cdots & -\frac{a_{2}}{a_{n-2}} & -\frac{a_{1}}{a_{n-2}} \\
0 & 0 & 0 & 0 & -\frac{a_{n-4}}{a_{n-3}} & \cdots & -\frac{a_{2}}{a_{n-3}} & -\frac{a_{1}}{a_{n-3}} \\
\vdots & \vdots & \vdots & \vdots & \vdots & \ddots & \vdots & \vdots \\
0 & 0 & 0 & 0 & 0 & \cdots & -\frac{a_{2}}{a_{3}} & -\frac{a_{1}}{a_{3}} \\
0 & 0 & 0 & 0 & 0 & \cdots & 0 & -\frac{a_{1}}{a_{2}}
\end{array}\right] .
$$

Now we find the lower triangular matrix $L$.

Let $I=\left[I_{1} ; I_{2} ; \cdots ; I_{n}\right]^{T}$ be the identity matrix of order $n$. Then the rows of the matrix $L=\left[l_{1} ; l_{2} ; \cdots ; l_{n}\right]^{T}$ are given by, $l_{j}=\left\{\begin{array}{l}I_{1}, j=1 \\ -\frac{1}{a_{n}} I_{1}+I_{2}, j=2 \\ -\frac{a_{n+3-j}}{a_{n+2-j}} I_{j-1}+I_{j}, j=3,4, \ldots, n,\end{array} \quad\right.$ which gives us that,

$$
\begin{gathered}
l_{1}=I_{1}=\left[\begin{array}{llllllll}
1 & 0 & 0 & 0 & \cdots & 0 & 0 & 0
\end{array}\right] . \\
l_{2}=-\frac{1}{a_{n}} I_{1}+I_{2}=\left[\begin{array}{llllllll}
-\frac{1}{a_{n}} & 1 & 0 & 0 & \cdots & 0 & 0 & 0
\end{array}\right] . \\
l_{3}=-\frac{a_{n}}{a_{n-1}} I_{2}+I_{3}=\left[\begin{array}{llllllll}
0 & -\frac{a_{n}}{a_{n-1}} & 1 & 0 & \cdots & 0 & 0 & 0
\end{array}\right] . \\
l_{4}=-\frac{a_{n-1}}{a_{n-2}} I_{3}+I_{4}=\left[\begin{array}{llllllll}
0 & 0 & -\frac{a_{n-1}}{a_{n-2}} & 1 & \cdots & 0 & 0 & 0
\end{array}\right] . \\
l_{5}=-\frac{a_{n-2}}{a_{n-3}} I_{4}+I_{5}=\left[\begin{array}{llllllll}
0 & 0 & 0 & -\frac{a_{n-2}}{a_{n-3}} & \cdots & 0 & 0 & 0
\end{array}\right] .
\end{gathered}
$$

Resuming like this until we get

$$
\begin{aligned}
l_{n-1} & =-\frac{a_{4}}{a_{3}} I_{n-2}+I_{n-1}=\left[\begin{array}{llllllll}
0 & 0 & 0 & 0 & \cdots & -\frac{a_{4}}{a_{3}} & 1 & 0
\end{array}\right] . \\
l_{n} & =-\frac{a_{3}}{a_{2}} I_{n-1}+I_{n}=\left[\begin{array}{llllllll}
0 & 0 & 0 & 0 & \cdots & 0 & -\frac{a_{3}}{a_{2}} & 1
\end{array}\right] .
\end{aligned}
$$

Consequently, the lower triangular matrix $L$ is 


$$
L=\left[\begin{array}{cccccccc}
1 & 0 & 0 & 0 & \cdots & 0 & 0 & 0 \\
-\frac{1}{a_{n}} & 1 & 0 & 0 & \cdots & 0 & 0 & 0 \\
0 & -\frac{a_{n}}{a_{n-1}} & 1 & 0 & \cdots & 0 & 0 & 0 \\
0 & 0 & -\frac{a_{n-1}}{a_{n-2}} & 1 & \cdots & 0 & 0 & 0 \\
0 & 0 & 0 & -\frac{a_{n-2}}{a_{n-3}} & \cdots & 0 & 0 & 0 \\
\vdots & \vdots & \vdots & \vdots & \ddots & \vdots & \vdots & \vdots \\
0 & 0 & 0 & 0 & \cdots & -\frac{a_{4}}{a_{3}} & 1 & 0 \\
0 & 0 & 0 & 0 & \cdots & 0 & -\frac{a_{3}}{a_{2}} & 1
\end{array}\right]
$$

\section{Bounds of the Zeros of Polynomials Based on the QR-Decomposition of C(p)}

In this section, we introduce some new bounds for the zeros of the complex monic polynomial $p(z)$. These bounds are based on the $Q R$-decomposition for the companion matrix $C(p)$.

Theorem 2 If $p(z)=z^{n}+a_{n} z^{n-1}+a_{n-1} z^{n-2}+\cdots+a_{2} z+a_{1}$ of degree $n \geq 2$ with $a_{1} \neq 0$ be a complex monic polynomial and let $z$ be a zero of $p(z)$ then

$$
|z| \leq\left[\sum_{i=0}^{n}\left|\frac{\mu_{i+1}}{\mu_{i}}\right|^{2}+\sum_{j=0}^{n-2} \sum_{i=2}^{n}\left|\frac{a_{i-1} \bar{a}_{n-j}}{\mu_{j} \mu_{j+1}}\right|^{2}\right]^{\frac{1}{2}}
$$

where $\mu_{k}=\sqrt{1+\sum_{j=1}^{k}\left|a_{n-j+1}\right|^{2}}$ for $k=1,2, \ldots, n-1, \mu_{0}=1$, and $\mu_{n}=\left|a_{1}\right|$.

Proof. The proof comes by noting the following inequalities,

$$
\begin{aligned}
|z| \leq & \left(\left[\begin{array}{cccccc}
-a_{n} & -a_{n-1} & -a_{n-2} & \cdots & -a_{2} & -a_{1} \\
1 & 0 & 0 & \cdots & 0 & 0 \\
0 & 1 & 0 & \cdots & 0 & 0 \\
\vdots & \vdots & \vdots & \ddots & \vdots & \vdots \\
0 & 0 & 0 & \cdots & 0 & 0 \\
0 & 0 & 0 & \cdots & 1 & 0
\end{array}\right]\right) \\
= & r(Q R) \leq\|Q R\|_{2} \\
= & \left\|\left[\begin{array}{cccccc}
\frac{\mu_{1}}{\mu_{0}} & \frac{a_{n-1} \bar{a}_{n}}{\mu_{0} \mu_{1}} & \frac{a_{n-2} \bar{a}_{n}}{\mu_{0} \mu_{1}} & \cdots & \frac{a_{2} \bar{a}_{n}}{\mu_{0} \mu_{1}} & \frac{a_{1} \bar{a}_{n}}{\mu_{0} \mu_{1}} \\
0 & \frac{\mu_{2}}{\mu_{1}} & \frac{a_{n-2} \bar{a}_{n-1}}{\mu_{1} \mu_{2}} & \cdots & \frac{a_{2} \bar{a}_{n-1}}{\mu_{1} \mu_{2}} & \frac{a_{1} \bar{a}_{n-1}}{\mu_{1} \mu_{2}} \\
0 & 0 & \frac{\mu_{3}}{\mu_{2}} & \cdots & \frac{a_{2} \bar{a}_{n-2}}{\mu_{2} \mu_{3}} & \frac{a_{1} \bar{a}_{n-2}}{\mu_{2} \mu_{3}} \\
\vdots & \vdots & \vdots & \ddots & \vdots & \vdots \\
0 & 0 & 0 & \cdots & \frac{\mu_{n-1}}{\mu_{n-2}} & \frac{a_{1} \bar{a}_{2}}{\mu_{n-1} \mu_{n-2}} \\
0 & 0 & 0 & \ldots & 0 & \frac{\mu_{n}}{\mu_{n-1}}
\end{array}\right]\right\|_{2}
\end{aligned}
$$




$$
\begin{aligned}
& =\left(\begin{array}{c}
\left|\frac{\mu_{1}}{\mu_{0}}\right|^{2}+\cdots+\left|\frac{\mu_{3}}{\mu_{2}}\right|^{2}+\left|\frac{a_{n-1} \bar{a}_{n}}{\mu_{0} \mu_{1}}\right|^{2}+\cdots+\left|\frac{a_{1} \bar{a}_{n}}{\mu_{0} \mu_{1}}\right|^{2}+\left|\frac{a_{n-2} \bar{a}_{n-1}}{\mu_{1} \mu_{2}}\right|^{2} \\
+\cdots+\left|\frac{a_{1} \bar{a}_{n-1}}{\mu_{1} \mu_{2}}\right|^{2}+\cdots+\left|\frac{a_{1} \bar{a}_{2}}{\mu_{n-1} \mu_{n-2}}\right|^{2}
\end{array}\right)^{\frac{1}{2}} \\
& =\left[\sum_{i=0}^{n}\left|\frac{\mu_{i+1}}{\mu_{i}}\right|^{2}+\sum_{j=0}^{n-2} \sum_{i=2}^{n}\left|\frac{a_{i-1} \bar{a}_{n-j}}{\mu_{j} \mu_{j+1}}\right|^{2}\right]^{\frac{1}{2}}
\end{aligned}
$$

Theorem 3 If $p(z)=z^{n}+a_{n} z^{n-1}+a_{n-1} z^{n-2}+\cdots+a_{2} z+a_{1}$ of degree $n \geq 2$ with $a_{1} \neq 0$ be a complex monic polynomial and let $z$ be a zero of $p(z)$ then

$$
\begin{gathered}
|z| \leq \max \left\{\left|\frac{\mu_{1}}{\mu_{0}}\right|,\left|\frac{\mu_{2}}{\mu_{1}}\right|+\left|\frac{a_{n-1} \bar{a}_{n}}{\mu_{0} \mu_{1}}\right|,\left|\frac{\mu_{3}}{\mu_{2}}\right|+\left|\frac{a_{n-2} \bar{a}_{n}}{\mu_{0} \mu_{1}}\right|+\left|\frac{a_{n-2} \bar{a}_{n-1}}{\mu_{1} \mu_{2}}\right|, \ldots,\right. \\
\left.\left|\frac{\mu_{n}}{\mu_{n-1}}\right|+\left|\frac{a_{1} \bar{a}_{n}}{\mu_{0} \mu_{1}}\right|+\left|\frac{a_{1} \bar{a}_{n-1}}{\mu_{1} \mu_{2}}\right|+\cdots+\left|\frac{a_{1} \bar{a}_{2}}{\mu_{n-1} \mu_{n-2}}\right|\right\}
\end{gathered}
$$

where $\mu_{k}=\sqrt{1+\sum_{j=1}^{k}\left|a_{n-j+1}\right|^{2}}$ for $k=1,2, \ldots, n-1, \mu_{0}=1$, and $\mu_{n}=\left|a_{1}\right|$.

Proof. The proof comes from the following inequalities

$$
\begin{aligned}
& |z| \leq r\left(\left[\begin{array}{cccccc}
-a_{n} & -a_{n-1} & -a_{n-2} & \cdots & -a_{2} & -a_{1} \\
1 & 0 & 0 & \cdots & 0 & 0 \\
0 & 1 & 0 & \cdots & 0 & 0 \\
\vdots & \vdots & \vdots & \ddots & \vdots & \vdots \\
0 & 0 & 0 & \cdots & 0 & 0 \\
0 & 0 & 0 & \cdots & 1 & 0
\end{array}\right]\right) \\
& =r(Q R) \leq\|Q R\|_{1} \\
& \|\left[\begin{array}{llllll}
\frac{\mu_{1}}{\mu_{0}} & \frac{a_{n-1} \bar{a}_{n}}{\mu_{0} \mu_{1}} & \frac{a_{n-2} \bar{a}_{n}}{\mu_{0} \mu_{1}} & \ldots & \frac{a_{2} \bar{a}_{n}}{\mu_{0} \mu_{1}} & \frac{a_{1} \bar{a}_{n}}{\mu_{0} \mu}
\end{array}\right. \\
& \mid \begin{array}{cccccc}
0 & \frac{\mu_{2}}{\mu_{1}} & \frac{a_{n-2} \bar{a}_{n-1}}{\mu_{1} \mu_{2}} & \ldots & \frac{a_{2} \bar{a}_{n-1}}{\mu_{1} \mu_{2}} & \frac{a_{1} \bar{a}_{n-1}}{\mu_{1} \mu_{2}}
\end{array} \\
& =\left\|\left[\begin{array}{cccccc}
0 & 0 & \frac{\mu_{3}}{\mu_{2}} & \ldots & \frac{a_{2} \bar{a}_{n-2}}{\mu_{2} \mu_{3}} & \frac{a_{1} \bar{a}_{n-2}}{\mu_{2} \mu_{3}} \\
\vdots & \vdots & \vdots & \ddots & \vdots & \vdots \\
0 & 0 & 0 & \ldots & \frac{\mu_{n-1}}{\mu_{n-2}} & \frac{a_{1} \bar{a}_{2}}{\mu_{n-1} \mu_{n-2}} \\
0 & 0 & 0 & \ldots & 0 & \frac{\mu_{n}}{\mu_{n-1}}
\end{array}\right]\right\|_{1} \\
& =|z| \leq \max \left\{\begin{array}{c}
\left|\frac{\mu_{1}}{\mu_{0}}\right|,\left|\frac{\mu_{2}}{\mu_{1}}\right|+\left|\frac{a_{n-1} \bar{a}_{n}}{\mu_{0} \mu_{1}}\right|,\left|\frac{\mu_{3}}{\mu_{2}}\right|+\left|\frac{a_{n-2} \bar{a}_{n}}{\mu_{0} \mu_{1}}\right|+\left|\frac{a_{n-2} \bar{a}_{n-1}}{\mu_{1} \mu_{2}}\right|, \\
\ldots,\left|\frac{\mu_{n}}{\mu_{n-1}}\right|+\left|\frac{a_{1} \bar{a}_{n}}{\mu_{0} \mu_{1}}\right|+\left|\frac{a_{1} \bar{a}_{n-1}}{\mu_{1} \mu_{2}}\right|+\cdots+\left|\frac{a_{1} \bar{a}_{2}}{\mu_{n-1} \mu_{n-2}}\right|
\end{array}\right\}
\end{aligned}
$$

Theorem 4 If $p(z)=z^{n}+a_{n} z^{n-1}+a_{n-1} z^{n-2}+\cdots+a_{2} z+a_{1}$ of degree $n \geq 2$ with $a_{1} \neq 0$ be a complex monic polynomial and let $z$ be a zero of $p(z)$ then

$$
|z| \leq \max \left\{\left|\frac{\mu_{n}}{\mu_{n-1}}\right|,\left|\frac{\mu_{n-1}}{\mu_{n-2}}\right|+\left|\frac{a_{1} \bar{a}_{2}}{\mu_{n-1} \mu_{n-2}}\right|, \ldots,\left|\frac{\mu_{1}}{\mu_{0}}\right|+\left|\frac{a_{n-1} \bar{a}_{n}}{\mu_{0} \mu_{1}}\right|+\left|\frac{a_{n-2} \bar{a}_{n}}{\mu_{0} \mu_{1}}\right|+\cdots+\left|\frac{a_{1} \bar{a}_{n}}{\mu_{0} \mu_{1}}\right|\right\}
$$


where $\mu_{k}=\sqrt{1+\sum_{j=1}^{k}\left|a_{n-j+1}\right|^{2}}$ for $k=1,2, \ldots, n-1, \mu_{0}=1$, and $\mu_{n}=\left|a_{1}\right|$.

Proof. The proof comes from the following inequalities

$$
\begin{aligned}
& |z| \leq r\left(\left[\begin{array}{cccccc}
-a_{n} & -a_{n-1} & -a_{n-2} & \cdots & -a_{2} & -a_{1} \\
1 & 0 & 0 & \cdots & 0 & 0 \\
0 & 1 & 0 & \cdots & 0 & 0 \\
\vdots & \vdots & \vdots & \ddots & \vdots & \vdots \\
0 & 0 & 0 & \cdots & 0 & 0 \\
0 & 0 & 0 & \cdots & 1 & 0
\end{array}\right]\right) \\
& =r(Q R) \leq\|Q R\|_{\infty}=\|R\|_{\infty} \\
& =\left\|\left[\begin{array}{cccccc}
\frac{\mu_{1}}{\mu_{0}} & \frac{a_{n-1} \bar{a}_{n}}{\mu_{0} \mu_{1}} & \frac{a_{n-2} \bar{a}_{n}}{\mu_{0} \mu_{1}} & \ldots & \frac{a_{2} \bar{a}_{n}}{\mu_{0} \mu_{1}} & \frac{a_{1} \bar{a}_{n}}{\mu_{0} \mu_{1}} \\
0 & \frac{\mu_{2}}{\mu_{1}} & \frac{a_{n-2} \bar{a}_{n-1}}{\mu_{1} \mu_{2}} & \ldots & \frac{a_{2} \bar{a}_{n-1}}{\mu_{1} \mu_{2}} & \frac{a_{1} \bar{a}_{n-1}}{\mu_{1} \mu_{2}} \\
0 & 0 & \frac{\mu_{3}}{\mu_{2}} & \ldots & \frac{a_{2} \bar{a}_{n-2}}{\mu_{2} \mu_{3}} & \frac{a_{1} \bar{a}_{n-2}}{\mu_{2} \mu_{3}} \\
\vdots & \vdots & \vdots & \ddots & \vdots & \vdots \\
0 & 0 & 0 & \ldots & \frac{\mu_{n-1}}{\mu_{n-2}} & \frac{a_{1} \bar{a}_{2}}{\mu_{n-1} \mu_{n-2}} \\
0 & 0 & 0 & \ldots & 0 & \frac{\mu_{n}}{\mu_{n-1}}
\end{array}\right]\right\|_{\infty} \\
& =\max \left\{\left|\frac{\mu_{n}}{\mu_{n-1}}\right|,\left|\frac{\mu_{n-1}}{\mu_{n-2}}\right|+\left|\frac{a_{1} \bar{a}_{2}}{\mu_{n-1} \mu_{n-2}}\right|, \ldots,\left|\frac{\mu_{1}}{\mu_{0}}\right|+\left|\frac{a_{n-1} \bar{a}_{n}}{\mu_{0} \mu_{1}}\right|+\left|\frac{a_{n-2} \bar{a}_{n}}{\mu_{0} \mu_{1}}\right|+\cdots+\left|\frac{a_{1} \bar{a}_{n}}{\mu_{0} \mu_{1}}\right|\right\}
\end{aligned}
$$

\section{Bounds of the Zeros of $p(z)$ Based on the LU Decomposition of $C(p)$}

Here in this section, we establish some new bounds for the zeros of the complex monic polynomial $\mathrm{p}(\mathrm{z})$. These bounds are depending on the LU decomposition for the companion matrix $\mathrm{C}(\mathrm{p})$ stated in section 2 above.

Theorem 5 If $p(z)=z^{n}+a_{n} z^{n-1}+a_{n-1} z^{n-2}+\cdots+a_{2} z+a_{1}$ of degree $n \geq 2$ with $a_{1} \neq 0$ be a complex monic polynomial and let $z$ be a zero of $p(z)$ then

$$
|z| \leq\left[\left(n+\frac{1}{\left|a_{n}\right|^{2}}\right)+\sum_{i=2}^{n-1}\left|\frac{a_{i+1}}{a_{i}}\right|^{2}\right]^{\frac{1}{2}}\left[\left(\sum_{i=1}^{n}\left|a_{i}\right|^{2}\right)+\left(\sum_{j=1}^{n-1} \frac{\sum_{i=1}^{j}\left|a_{j}\right|^{2}}{\left|a_{j+1}\right|^{2}}\right)\right]^{\frac{1}{2}}
$$

Proof. The proof comes from the following inequalities

$$
\begin{aligned}
|z| \leq & r(C)=r(L U) \leq\|L U\|_{2} \leq\|L\|_{2}\|U\|_{2} \\
= & {\left[1+1+\cdots+1+\left|\frac{1}{a_{n}}\right|+\left|\frac{a_{3}}{a_{2}}\right|+\cdots+\left.\left|\frac{a_{n}}{a_{n-1}}\right|\right|^{\frac{1}{2}}\right.} \\
& {\left[\begin{array}{c}
\left.\left|a_{n}\right|^{2}+\cdots+\left|a_{1}\right|^{2}+\left|\frac{a_{n-1}}{a_{n}}\right|^{2}+\cdots+\left|\frac{a_{1}}{a_{n}}\right|^{2}+\left|\frac{a_{n-2}}{a_{n-1}}\right|^{2}\right]^{\frac{1}{2}} \\
+\cdots+\left|\frac{a_{1}}{a_{n-1}}\right|^{2}+\cdots+\left|\frac{a_{2}}{a_{3}}\right|^{2}+\left|\frac{a_{1}}{a_{3}}\right|^{2}+\left|\frac{a_{1}}{a_{2}}\right|^{2}
\end{array}\right] } \\
= & {\left[\left(n+\frac{1}{\left|a_{n}\right|^{2}}\right)+\sum_{i=2}^{n-1}\left|\frac{a_{i+1}}{a_{i}}\right|^{2}\right]^{\frac{1}{2}}\left[\left(\sum_{i=1}^{n}\left|a_{i}\right|^{2}\right)+\left(\sum_{j=1}^{n-1} \frac{\sum_{i=1}^{j}\left|a_{j}\right|^{2}}{\left|a_{j+1}\right|^{2}}\right)\right]^{\frac{1}{2}} }
\end{aligned}
$$


Theorem 6 If $p(z)=z^{n}+a_{n} z^{n-1}+a_{n-1} z^{n-2}+\cdots+a_{2} z+a_{1}$ of degree $n \geq 2$ with $a_{1} \neq 0$ be a complex monic polynomial and let $z$ be a zero of $p(z)$ then

$$
\begin{aligned}
|z| \leq & \max \left\{1,1+\left|\frac{1}{a_{n}}\right|, 1+\left|\frac{a_{3}}{a_{2}}\right|, \ldots, 1+\left|\frac{a_{n}}{a_{n-1}}\right|\right\} \\
\left|a_{n}\right|,\left|a_{n-1}\right|\left(1+\left|\frac{1}{a_{n}}\right|\right),\left|a_{n-2}\right|\left(1+\left|\frac{1}{a_{n}}\right|+\left|\frac{1}{a_{n-1}}\right|\right), & \max \left\{\begin{array}{c}
\left|a_{n-3}\right|\left(1+\left|\frac{1}{a_{n}}\right|+\left|\frac{1}{a_{n-1}}\right|+\left|\frac{1}{a_{n-2}}\right|\right), \ldots,\left|a_{1}\right|\left(1+\left|\frac{1}{a_{n}}\right|+\left|\frac{1}{a_{n-1}}\right|+\cdots+\left|\frac{1}{a_{2}}\right|\right)
\end{array}\right\}
\end{aligned}
$$

Proof. The proof comes from the following inequalities

$$
\begin{aligned}
|z| \leq & r(C) \\
= & r(L U) \leq\|L U\|_{2} \\
\leq & \|L\|_{2}\|U\|_{2} \\
= & \max \left\{1,1+\left|\frac{1}{a_{n}}\right|, 1+\left|\frac{a_{3}}{a_{2}}\right|, \ldots, 1+\left|\frac{a_{n}}{a_{n-1}}\right|\right\} \\
& \max \left\{\begin{aligned}
\left|a_{n}\right|,\left|a_{n-1}\right|\left(1+\left|\frac{1}{a_{n}}\right|\right),\left|a_{n-2}\right|\left(1+\left|\frac{1}{a_{n}}\right|+\left|\frac{1}{a_{n-1}}\right|\right), \\
\left|a_{n-3}\right|\left(1+\left|\frac{1}{a_{n}}\right|+\left|\frac{1}{a_{n-1}}\right|+\left|\frac{1}{a_{n-2}}\right|\right), \ldots,\left|a_{1}\right|\left(1+\left|\frac{1}{a_{n}}\right|+\left|\frac{1}{a_{n-1}}\right|+\cdots+\left|\frac{1}{a_{2}}\right|\right)
\end{aligned}\right\}
\end{aligned}
$$

Theorem 7 If $p(z)=z^{n}+a_{n} z^{n-1}+a_{n-1} z^{n-2}+\cdots+a_{2} z+a_{1}$ of degree $n \geq 2$ with $a_{1} \neq 0$ be a complex monic polynomial and let $z$ be a zero of $p(z)$ then

$$
\begin{aligned}
|z| \leq & \max \left\{1,1+\left|\frac{1}{a_{n}}\right|, 1+\left|\frac{a_{3}}{a_{2}}\right|, \ldots, 1+\left|\frac{a_{n}}{a_{n-1}}\right|\right\} \\
& \max \left\{\sum_{i=1}^{n}\left|a_{i}\right|, \frac{1}{\left|a_{n}\right|} \sum_{i=1}^{n-1}\left|a_{i}\right|, \frac{1}{\left|a_{n-1}\right|} \sum_{i=1}^{n-2}\left|a_{i}\right|, \ldots, \frac{\left|a_{1}\right|+\left|a_{2}\right|}{\left|a_{3}\right|}, \frac{\left|a_{1}\right|}{\left|a_{2}\right|}\right\}
\end{aligned}
$$

Proof. The proof comes from the following inequalities

$$
\begin{aligned}
|z| \leq & r(C) \\
= & r(L U) \leq\|L U\|_{2} \\
\leq & \|L\|_{2}\|U\|_{2} \\
= & \max \left\{1,1+\left|\frac{1}{a_{n}}\right|, 1+\left|\frac{a_{3}}{a_{2}}\right|, \ldots, 1+\left|\frac{a_{n}}{a_{n-1}}\right|\right\} \\
& \max \left\{\sum_{i=1}^{n}\left|a_{i}\right|, \frac{1}{\left|a_{n}\right|} \sum_{i=1}^{n-1}\left|a_{i}\right|, \frac{1}{\left|a_{n-1}\right|} \sum_{i=1}^{n-2}\left|a_{i}\right|, \ldots, \frac{\left|a_{1}\right|+\left|a_{2}\right|}{\left|a_{3}\right|}, \frac{\left|a_{1}\right|}{\left|a_{2}\right|}\right\}
\end{aligned}
$$

\section{Acknowledgements}

The authors would like to thank Professor Fuad Kittaneh for his spirit and valuable advices that lead to complete this work.

\section{References}

Bhatia, R. (1997). Matrix Analysis. New York: Springer-Verlage. http://dx.doi.org/10.1007/978-1-4612-0653-8

Horn, R. A., \& Johnson, C. R. (1985). Matrix Analysis. Cambridge: Cambridge University Press. http://dx.doi.org/10.1017/CBO9780511810817

Horn, R. A., \& Johnson, C. R. (1991). Topics in Matrix Analysis. Cambridge: Cambridge University Press. http://dx.doi.org/10.1017/CBO9780511840371 
Issa, A. S. (2009). Master Thesis. Not published.

Kittaneh, F. (2003). Bounds for the Zeros of Polynomials from Matrix Inequalities. Arch. Math. (Basel), 81, 601-608. Retrieved from http://link.springer.com/article/10.1007\%2Fs00013-003-0525-6

Kittaneh, F., \& Shabrawi, K. (2006). Some Decomposition Results for Companion Matrices. J. Math. Anal. Appl, 318, 626-636. http://dx.doi.org/10.1016/j.jmaa.2005.05.071

Kittaneh, F., \& Shabrawi, K. (2007). Bounds for the Zeros of Polynomials from Matrix Inequalities II. Linear and Multilinear Algebra, 55, 147-158. http://dx.doi.org/10.1080/03081080600665481

Marden, M. (1969). Geometry of Polynomials (2nd ed.). Amer. Math. Surveys, providence, RI.

Mayer, C. D. (2000). Matrix Analysis and Applied Linear Algebra. SIAM. http://dx.doi.org/10.1137/1.9780898719512

\section{Copyrights}

Copyright for this article is retained by the author(s), with first publication rights granted to the journal.

This is an open-access article distributed under the terms and conditions of the Creative Commons Attribution license (http://creativecommons.org/licenses/by/3.0/). 